\title{
NEUROTRANSMITTERS AND CARDIOVASCULAR RESPONSES TO AEROBIC AND RESISTANCE EXERCISE IN MEN ADDICTED TO METHAMPHETAMINE
}

\author{
Hamid Arazi', Seyedeh Shiva Dadvand ${ }^{1}$, Mehnoush Tavakoli Fard ${ }^{2}$ \\ University of Guilan', Rasht, Iran \\ Addiction Research and Treatment institute of Hayateno ${ }^{2}$, Rasht, Iran
}

\begin{abstract}
Background. This study aimed to examine serotonin, dopamine and cardiovascular responses to aerobic and resistance exercise in men addicted to methamphetamine during rehabilitation.

Methods. Subjects were 10 men addicted to methamphetamine with an average age of $31.2 \pm 6.2$ years old, height: $173 \pm 5.6 \mathrm{~cm}$, weight: $73.66 \pm 12.5 \mathrm{~kg}$. In the first session the subjects were acquainted with the environment. The variables measured at the second session were physical and physiological characteristics. In the third and fourth sessions, projects to ensure the effectiveness of the pilot were conducted. In the fifth and sixth sessions which were spaced 7 days apart, aerobic and resistance exercises were carried out. The variables which were measured before and after exercise programs included heart rate, blood pressure and circulatory levels of serotonin and dopamine.

Results. The increase in blood serotonin and dopamine levels after both aerobic and resistance exercise were significant $(p<.05)$, but neither of the two aerobic and resistance exercise were superior to each other. In addition, decrease in systolic and diastolic blood pressure, heart rate, mean arterial pressure, pulse pressure and myocardial oxygen cost at some time intervals after these two exercises were significant $(p<.05)$, However, there were no significant changes in comparison of exercises $(p>.05)$.

Conclusions. Both of these exercises can be used as valuable support factors in treatment of addiction.
\end{abstract}

Keywords: physical activity, methamphetamine, neurotransmitter, blood pressure.

\section{INTRODUCTION}

$\mathrm{A}$ mong various types of drugs, methamphetamine is a highly addictive stimulant and is widely abused. In 1893, for the first time synthesized methamphetamine was used to treat hyperactivity, narcolepsy, asthma and obesity (Varner, Ogden, Delcarpio, \& MelegSmith, 2002; Zweben et al., 2004). Stimulants are substances that increase the activity of the central nervous system and generate increased heart rate and blood pressure. As a result, methamphetamine enhances awareness, creates a sense of confidence and energy, increases concentration and conscious behaviour. Chronic methamphetamine abuse reduces dopamine transport in the striatum and to a lesser extent in the frontal cortex of the consumers, produces depletion of dopamine terminals in the brain (McCann et al., 2008). On the contrary, high doses of methamphetamine also cause depletion of serotonin terminals (Ricaurte, Schuster, \& Seiden, 1980). In fact, psycho-stimulants not only inhibit the transmitters of dopamine and increase the release of dopamine (Seiden, Sabol, \& Ricaurte 1993), but also inhibit serotonin reuptake and increase it in extracellular space of the cell (Andrews \& Lucki, 2001). In a study, Thompson et al. (2004) compared the methamphetamine-dependent patients and healthy subjects and concluded that long-term use of methamphetamine damaged dopaminergic, serotonergic and metabolism systems of the brain. The results showed that the brains of its consumers 
had structural anomalies. Based on the results reported by Harvey, Lacan, Tanious, \& Melega, (2000), damage caused by drug use in dopaminergic system needs 12 to 17 months for recovery, and the time for serotonergic system is 6 months. A steady state concentration of norepinephrine in the brain of the rat race- Sprague after 8 weeks of interval training program was found by Brown and Van Huss (1973). The expanded findings were explored by checking norepinephrine and serotonin in three regions of the brain (cortex, cerebellum, and remainder of the brain cerebral) of sedentary female rats (Brown et al., 1979). Both norepinephrine and serotonin levels in many brain regions of trained rats were significantly higher. As Spirduso and Farrar (1981) showed, endurance activity in aging rats was weakened, and movement imitation was reduced. As it was shown, movement imitation was connected with the work of nigrostriatal dopamine, and it seems reasonable to suggest that regular physical exercise may directly or indirectly cause changes in the nigrostriatal dopamine. Many neurotransmitters such as dopamine, glutamate, acetylcholine, serotonin, GABA (gamma amino butyric acid) and endogenous opioids (Floresco, Blaha, Yang, \& Phillips, 2001) play a role in the development of drug dependency and addiction. Methamphetamine consumption can create a variety of cardiovascular problems including increased heart rate, arrhythmia, hypertension and myocardial infarction (Varner et al., 2002). Addiction treatment was in terms of psychological aspects in the past, but later treatments were replaced by medical therapies (Wendt, 2002). Due to the limited studies on the effects of exercise on treatment and addiction, researchers conducted the current study. Treatment process along with conventional drug therapy, strengthening exercise on serotonin, dopamine levels as factors affected by addiction and, most importantly, the ambiguity in research on common sports activities to promote and protect health encouraged the researchers to investigate serotonin, dopamine and cardiovascular responses to aerobic and resistance exercise in men addicted to methamphetamine during rehabilitation.

\section{METHODS}

Participants. This study was a quasiexperimental study conducted on men addicted to methamphetamine at "Tarannom Ehyaye No" Institute in the city of Rasht. The subjects' inclusion criteria to this study were a lack of musculoskeletal lesions, no record of mental, cardiovascular, thyroid, gastrointestinal, diabetes, AIDS and hepatitis illnesses, no consumption of drugs and cigarettes, or a lack of physical activity in the past month. At the institute of addiction, people were under medical supervision, and they were selected according to the blood samples analysed and laboratory results checked by physicians. Ten addicted men with an average age of $31.2 \pm 6.2$ years, height $1.73 \pm 0.56 \mathrm{~m}$, weight $73.66 \pm 12.5 \mathrm{~kg}$, BMI $24.34 \pm 3.03 \mathrm{~kg} / \mathrm{m}^{2}$ and $\mathrm{VO}_{2} \max 30.77 \pm 2.53 \mathrm{ml} / \mathrm{kg} / \mathrm{min}$ were chosen purposefully. After administering a consent form and a questionnaire for stress management, participants took part in aerobic and resistance exercise program. In order to precisely control the daily diet of the subjects, the diet record form was used. Nutritional assessment was based on 24 hour recall questionnaire. Questionnaires were evaluated based on 3 days a week. It was found that in addition to the same diet, average daily calorie uptake including carbohydrate, fat and protein was not significantly different. Throughout the day, participants had the same type of meals. Validated food frequency questionnaire for nutrients and food groups was used in the same way as in previous studies. Participants also were recommended not to engage in any strenuous physical activity for 48 hours before the test and to avoid taking too much salt. In the first session the subjects were acquainted with the environment and the way to run the test. The variables measured at the second session were physical characteristics such as age, weight, height, body mass index, waist to hip ratio, body fat percentage and physiological variables such as muscular strength and endurance. In the third and fourth sessions, aiming to ensure the effectiveness of the type, duration and intensity of exercise and cardiorespiratory endurance test, the pilot studies were conducted. In the fifth and sixth sessions with an interval of 7 days between them, aerobic and resistance exercises were carried out. Similarly, before and immediately after the implementation of the exercise program, (aerobic and resistance) variables such as serotonin, dopamine, heart rate, blood pressure (systolic, diastolic) and myocardial oxygen were measured. Intervals of $15,30,45$ and 60 minutes after the exercises were measured and recorded. The procedures were approved by the Institutional Ethics Review Committee of the University. 
Procedure. The height of the subjects was measured using a stadiometer (made in Iran with an accuracy of one millimetre) and weight was measured using digital scales (Model Seca, South Korea, with a sensitivity of $0.1 \mathrm{~kg}$ ). After measuring the height and weight of the subjects the Body Mass Index (BMI) was calculated by dividing weight $(\mathrm{kg})$ by the square of height $\left(\mathrm{m}^{2}\right)$. In order to determine the amount of body fat, subcutaneous fat in the areas of the chest, abdomen and thigh on the right side of the body was measured with callipers YAGAMI (model Eiyoken type, Japan), and it was calculated using Jackson and Pollock equation which is especially developed for men (Lohman et al., 1991).

Cardiorespiratory endurance test was conducted during one mile walking using a chronometer and pulse meter (Polar model, Finland). Subjects walked the distance at their desired speed. By measuring the time and their heart rate at the end of the determined distance, the maximum oxygen consumption was estimated (George et al., 1993) using the following formula:

$\mathrm{VO}_{2} \max =100.5+(8.344 \times \operatorname{sex})-(0.1636 \times$ $\mathrm{Kg})-(1.438 \times$ time $)-(0.1928 \times \mathrm{HR})$

Notes. Gender: Male 0, Female 1; Weight $=\mathrm{Kg}$; time $=$ time for jogging a mile; $\mathrm{HR}=$ heart rate.

Blood pressure measurement was done using mercury sphygmomanometer and stethoscope (model ALPK2, Japan). In order to measure the subjects' heart rate Beurer Stethoscope (model PM80, Germany) was used. Following the measurement of resting systolic blood pressure, the heart rate of subjects, and the amount of myocardial oxygen expenditure was estimated using the following formula (Kenney et al., 2015):

Myocardial oxygen expenditure $=100 /$

$(\mathrm{HR} \times \mathrm{SBP})$

In addition, the mean arterial pressure was calculated using the following formula:

Mean arterial pressure $=$ Diastolic pressure + 1.3 pulse pressure. To measure upper body muscular endurance, Swedish push-up was used and the number of correct moves in one minute was recorded. To determine the strength of the participants (in the movements) Brzycki formula was used (Brzycki, 1993):

$$
1 \mathrm{RM}=\frac{\text { weight }(\mathrm{kg})}{1.0278-0.0278 \times \text { number of repetitions }}
$$

Exercise protocols. Aerobic exercise protocol consisted of three phases: warm up, the main part and the cool down for 45 minutes. Warm up involved jogging, stretching and flexibility in the upper and lower limbs for 10 minutes. During the main exercise, individuals ran two turns in 15 minutes with an intensity of $75-70 \%$ of maximum heart rate, resting at intervals of 3 to 4 in minutes between the stages. Finally, cooling down with stretching and flexibility exercises and relaxation was carried out for 5 minutes. Moreover, the maximum heart rate was calculated using the following formula (Tanaka et al., 2001):

Maximum heart rate $=208-(0.7 \times$ age of the subject)

Resistance exercise protocol within one week of aerobic protocol implementation consisted of three phases: warm up, the main part, and the cool down (about 45 minutes) in which warmingup and cooling-down phases of the exercises were similar to aerobic exercise phases. In the main part, individuals generally performed four exercises (bench press, leg extension, leg flexion, rowing). Two sets per exercise included 10 repetitions and rest intervals of 2-3 minutes. At the end, cooling activities like aerobic exercise were performed for 5 minutes.

Blood sampling. To determine the blood levels of serotonin and dopamine before and after aerobic and resistance exercise (with one week break between the protocols), blood was withdrawn. Following a 12 hours of fasting at night, $5 \mathrm{ml}$ blood was taken from brachial vein of the subjects in the sitting position at $10 \mathrm{am}$ in the presence of a specialist. The blood sampling was repeated immediately after the exercises and it was placed into dry sterile tubes and sent to the laboratory. The blood was centrifuged at 3000 RPM for 15 minutes. At this point to measure the variables, serum was separated from plasma and was frozen in $-70^{\circ} \mathrm{C}$ temperature. In order to determine serotonin and dopamine of plasma, ELISA and kit LDN method (Germany), the coefficient of variation of dopamine intra assay 22.5 and inter assay 14.2; serotonin intra assay 9.7 and inter assay 10.4 and Device STAT FAX (Made in USA) were used.

Statistical analysis. Normality of the data was checked using the Kolmogorov-Smirnov normality test. A $2 \times 6$ factorial analysis of variance was conducted with repeated measures (Aerobic and Resistance protocols x blood pressure; and heart rate measurements before and immediately after exercise and at T15, T30, T45, and T60). Posthoc multiple comparisons with correction of the 
confidence interval were made using Bonferroni method. A 2 × 2 factorial analysis of Independent and Paired Sample $t$ Test measures (Aerobic and Resistance protocols $\mathrm{x}$ blood levels of serotonin and dopamine measurements at before and after exercise programs) were performed. For data analysis, SPSS version 20 was used and significance level was set at $p<.05$.

\section{RESULTS}

Characteristics of subjects are shown in Table 1.

Table 1. The mean and standard deviation of the primary characteristics of subjects

\begin{tabular}{|c|c|}
\hline Characteristics & Mean \pm SD \\
\hline Age (years) & $32.22 \pm 6.2$ \\
\hline Weight $(\mathrm{kg})$ & $73.6 \pm 12.5$ \\
\hline Height $(\mathrm{cm})$ & $173 \pm 5.6$ \\
\hline BMI $\left(\mathrm{kg} / \mathrm{m}^{2}\right)$ & $24.3 \pm 3.06$ \\
\hline Body fat $(\%)$ & $25.01 \pm 4.7$ \\
\hline Waist-to-hip ratio $(\mathrm{WHR})$ & $0.91 \pm 0.04$ \\
\hline VO ${ }_{2}$ max $(\mathrm{ml} / \mathrm{kg} / \mathrm{min})$ & $30.77 \pm 2.53$ \\
\hline Muscular endurance () & $21.8 \pm 4.3$ \\
\hline 1 RM bench press $(\mathrm{kg})$ & $34 \pm 6.7$ \\
\hline
\end{tabular}

Note. $\mathrm{SD}$ - standard deviation; $\mathrm{BMI}$ - Body Mass Index; $\mathrm{VO}_{2} \max -$ Maximal Oxygen Consumption, 1RM - One Repetition Maximum.

Serotonin and dopamine Table 2 shows the values of dopamine and serotonin before and after exercise. A significant increase in the levels of dopamine and serotonin was observed in both aerobic and resistance exercises $(p<.05)$. The mean difference between the dopamine and serotonin before and after aerobic and resistance exercise was not significantly different $(p<.05)$. Effect of both exercises on blood levels of serotonin and dopamine was the same. Considering these two mediators, none of the above mentioned exercises had much advantage over another.
Figure 1 demonstrates systolic and diastolic blood pressure and mean arterial pressure in time intervals before the exercise and immediately, 15 , 30,45 and 60 minutes after exercise. In aerobic exercise, systolic blood pressure after exercise and during 30 to 60 minutes of exercise had a significant difference compared to resting time $(p<.05)$. On the contrary, systolic blood pressure did not demonstrate a significant difference 15 minutes after exercise $(p>.05)$. Additionally, in resistance exercise there was a significant difference in systolic blood pressure immediately, 45 and 60 minutes after exercise to resting time $(p<.05)$. However, in this type of exercise, 15 minutes after exercise there was no significant difference $(p>.05)$. According to Figure 1, there was no significant difference between the effects of aerobic and resistance exercises on systolic pressure in different time phases $(p>.05)$.

Figure 1 shows immediate significant change in diastolic blood pressure in phases and 60 minutes after aerobic exercise compared to the resting time $(p<.05)$. In contrast, 15 to 45 minutes after aerobic exercise, there was no significant difference in diastolic pressure $(p>.05)$. Furthermore, in resistance exercise, changes in diastolic pressure immediately after the exercise were significant $(p<.05)$, nevertheless at time intervals of 15 to 60 minutes after the exercise, there was no significant change observed compared to the resting time. Also, between aerobic and resistance exercises, there was no significant change observed in diastolic pressure after exercise $(p>.05)$. Mean arterial pressure showed significant changes during aerobic exercises and immediately after exercise. This occurred at 45 and 60 minutes, and during resistance exercise immediately and 60 minutes after exercise $(p<.05)$. And vice versa, at 15,30 and 45 time intervals after exercise there was no significant change observed. Also, changes were not significant $(p>.05)$ in the mean arterial pressure of the groups.

\begin{tabular}{|c|c|c|c|c|}
\hline Variable & Exercise & Before & After & $\boldsymbol{p}$ \\
\hline \multirow{2}{*}{$\begin{array}{c}\text { Dopamine } \\
(\mathbf{p m g} / \mathbf{m l})\end{array}$} & aerobic & $78.8 \pm 5.5$ & $86 \pm 2.3$ & \multirow{2}{*}{.147} \\
\cline { 2 - 4 } & resistance & $83.88 \pm 7.7$ & $92.11 \pm 2.8$ & \\
\hline \multirow{2}{*}{$\begin{array}{c}\text { Serotonin } \\
(\mathbf{n g} / \mathbf{m l})\end{array}$} & aerobic & $63.5 \pm 17.5$ & $384.11 \pm 80$ & \multirow{2}{*}{.741} \\
\cline { 2 - 4 } & resistance & $86 \pm 16$ & $449 \pm 42$ & \\
\hline
\end{tabular}

Table 2. Comparing the mean difference between the dopamine and serotonin before and after aerobic and resistance exercise

Note. $*_{-}$indicates a significant difference between the aerobic and resistance exercises at level of $p<.05$. 
A

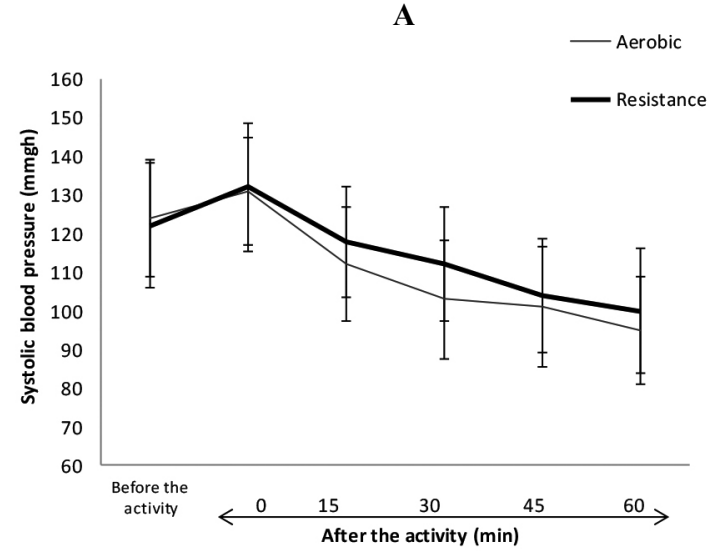

B

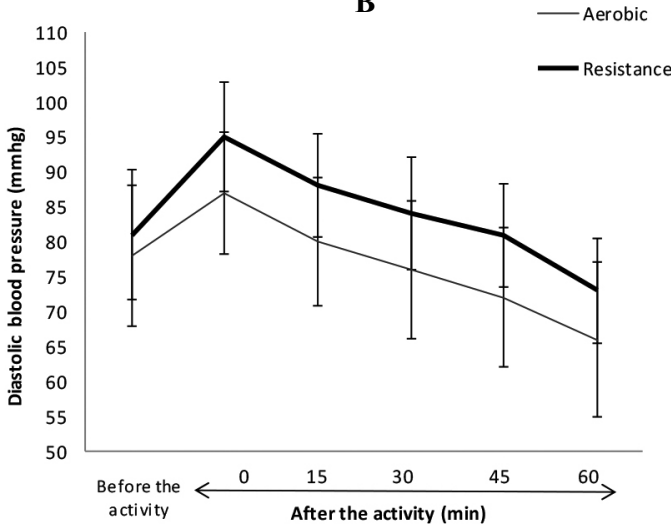

C

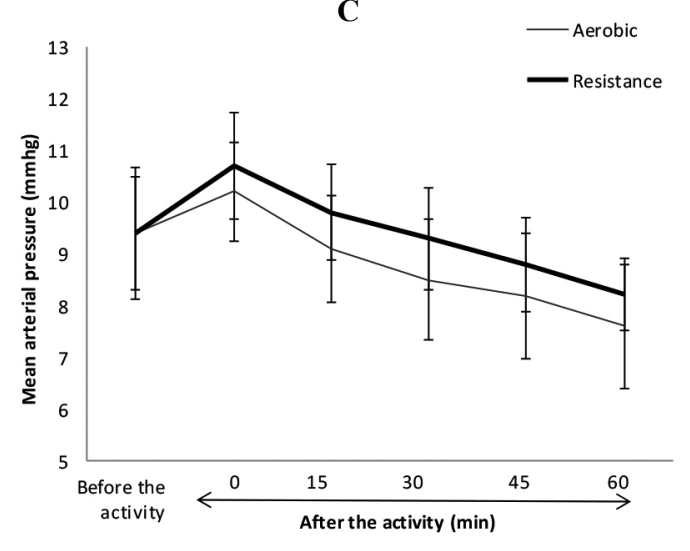

Figure 1. Changes in systolic blood pressure (A), diastolic (B) and mean arterial pressure (C) at intervals before, immediately, 15, 30, 45 and $60 \mathrm{~min}$ after aerobic and resistance exercise

Notes. \# - Significantly different in comparison with pre- resistance exercise. * - Significantly different in comparison with pre- aerobic exercise.

D

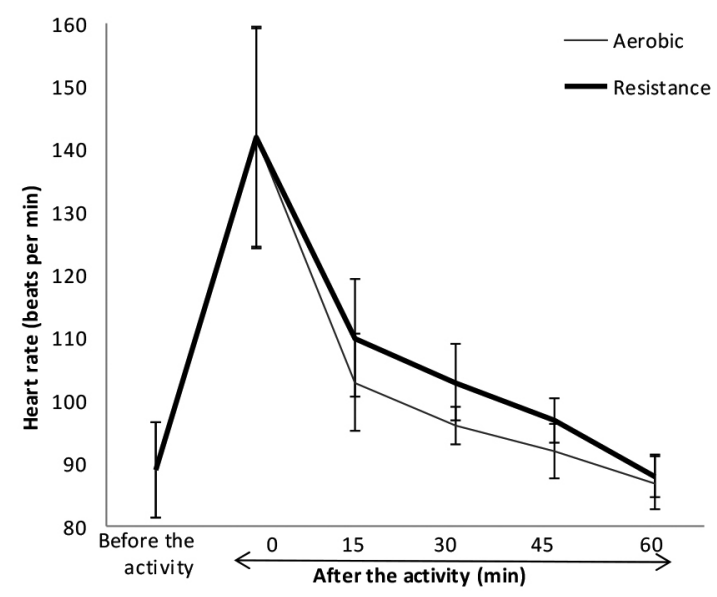

E

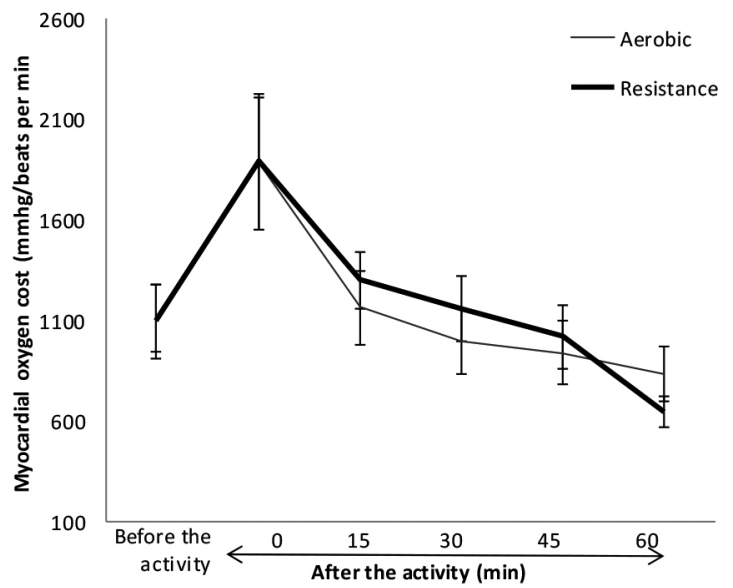

Figure 2. Changes in heart rate (D), and myocardial oxygen cost (E), at intervals before, immediately, 15, 30, 45 and 60 min after aerobic and resistance exercise

Notes. \#-Significantly different in comparison with pre- resistance exercise. ${ }^{*}$ - Significantly different in comparison with pre- aerobic exercise. 
Heart rate. Figure 2 shows that changes in heart rate immediately after exercise to resting time in aerobic session were significant $(p<.05)$, in addition, 15 to 60 minutes after exercise there was no significant change $(p>.05)$. Heart rate changes in resistance exercise immediately and 15 minutes after exercise were significant, nevertheless at 30 , 45 and 60 minutes after exercise, these changes were not significant $(p>.05)$. Changes in the heart rate were not significant in aerobic and resistance exercise $(p>.05)$.

Myocardial oxygen expenditure. According to Figure 2, changes in myocardial oxygen expenditure at time intervals immediately and 60 minutes after exercise were significant in aerobic exercise $(p<.05)$. Changes in myocardial oxygen expenditure at time intervals immediately, 15 and 60 minutes after exercise were significant in resistance exercise $(p<.05)$; nonetheless at other times there was no significant change $(p>.05)$. Changes in the expenditure of oxygen consumption were not significant in aerobic and resistance exercise $(p>.05)$.

\section{DISCUSSION}

The purpose of this study was to examine serotonin, dopamine and cardiovascular responses to aerobic and resistance exercise of men addicted to methamphetamine during rehabilitation. The findings suggest that aerobic and resistance exercise can cause a significant increase in blood levels of dopamine and serotonin with positive changes in the cardiovascular behaviour. In this regard, Mota et al. (2009) showed that eight weeks of aerobic and resistance exercise improved health and physical performance in men addicted to methamphetamine. Researchers stated that physical activity improved excitement, discomfort and symptoms related to methamphetamine through the effects of the endogenous opioid system and strengthening of the dopaminergic transmission. In addition, it was shown that exercise affected imaging techniques of the nervous system by effecting available receptors of $\mathrm{D} 2 / \mathrm{D} 3$ in methamphetamine consumers who were in the process of quitting. The results of Goekint et al. (2012) showed that aerobic exercise increased the dopamine levels. Also Alberghina, Giannetto, \& Piccione, (2010) and Langfort et al. (2006) suggested in their research that aerobic exercise could have a positive effect on serotonin level. Exercise resulted in the release of certain nerve messengers in the brain that diminish physical and mental pain. Most research conducted in this area focused on running, however, all aerobic exercises had such benefits. It has been found that the effects on the brain are applied through several mechanisms, including neuro-genesis, increasing patients' mood and the release of endorphins. Evidence suggests that exercise can change the release of many neurotransmitters such as dopamine, glutamate, acetylcholine, serotonin and endogenous opiate in the brain (Joanna et al., 2011). FontesRibeiro et al. (2011) pointed out that regular exercise had an overlap with drug abuse, therefore, the effect of exercise on the dopaminergic system, and dopamine changes was observed. The use of amphetamines causes lasting changes in brain reward system, accumbency area, and other areas in the brain. In addition, there is a relationship between dopamine and all behavioural aspects like physical activity. It has been proven that exercise increases the release of dopamine synthesis, neuroplasma stimulation, promoting health level and sense of well-being (Dey, Singh, \& Dey, 1992). O'dell Galvez, Ball, and Marshall (2012) examined the effects of exercise in improving the damage of dopaminergic and serotonergic terminals in mice addicted to methamphetamine. It was stated in their report that repeating the moderate dose of methamphetamine decreased the dopaminergic and serotonergic terminals as well as their receivers and enzymes. It was suggested that due to increased dopamine and serotonin voluntary exercise could be used in improving the damage resulting from drug abuse as a non-drug therapy (O'dell et al., 2012). Unlike the results of the present study, Dwyer and Flynn (2002) found that short term aerobic exercise in young men would not change the central sensitivity of serotonin receptors. In this study, the subjects had a 30 minutes bike riding with $70 \%$ of their maximum aerobic capacity for 3 times. The results showed that no significant changes were found in the regional sensitivity of serotonin receptors. It seems that in this research, exercise movements were not enough to make changes. Also, the findings of Wang et al. (2000) showed that 30 minutes of intense exercise on a treadmill had no effect on the dopamine rate of men and women. It seems that the reason for this lack of effect is the training of the subjects. The rate of dopamine increase in trained subjects was lower. The results indicate that aerobic and resistance exercise lowered systolic and diastolic blood pressure, heart rate, mean arterial pressure and myocardial oxygen cost in men addicted to methamphetamine. Despite some inconsistencies in 
the results of studies in blood pressure responses after exercise, most studies have shown that blood pressure is reduced after one session of resistance or acute resistance exercise to resting values before activity so that systolic blood pressure falls between 10 and $20 \mathrm{mmHg}$ per minutes and after about 2 to 3 minutes returns to amounts before activity. However, it takes about 10 minutes for the regulation of normal blood pressure by homeostasis systems (Mohebbi, Rahmani-Nia, Vatani, \& Faraji, 2010). Also, the study of Mota et al. (2009) showed that resistance and endurance activities reduce blood pressure, which is in consistent with the findings of the study. The subjects participated in two separate sessions of 20 minutes running on the treadmill at intensity rate of 70 to 80 percent of heart rate reserve and 20 minutes of circular resistance exercise with $40 \%$ of one repetition maximum. The results showed a decrease in systolic blood pressure after each activity (this reduction lasted longer after resistance exercise). Moreover, diastolic blood pressure reduction was observed only after resistance exercise and 30 minutes. The expenditure of myocardial oxygen after resistance exercise was higher than that of endurance activity due to more cardiovascular pressure compared to resistance exercise (Mota et al., 2009). The results of Dolezal et al. (2014) showed that physical activity improved the heart rate in those taking methamphetamine, which is in line with the results of this study. The researchers also suggest that physical activity may improve symptoms of cardiovascular disease and the effects of stress and help people manage stress in their life (Zweben et al., 2004). As one of the effects of endurance and resistance exercises, we can name cardiovascular responses which affect heart rate and heart rate variability (HRV). Studies have shown that exercise causes sinusoidal bradycardia in resting conditions in methamphetamine consumer groups and reduces the heart rate at submaximal oxygen consumption(Dolezal etal.,2014). Methamphetamine impact on catecholamines in the peripheral nervous system (such as noradrenaline and dopamine), also through its primary mechanism (heart rate and blood pressure) affects cardiovascular system (Karch $\&$ Drummer, 2001). Contrary to the findings of this study, the results of Jones, George, Edwards, and Atkinson (2007) showed that endurance exercise with 40 and 70 percent of maximal oxygen uptake has no significant effect on the hypotension. Also, Williams et al. (2007) showed that systolic, diastolic and mean blood pressure, heart rate, cardiac output, oxygen consumption and myocardial oxygen cost are increased in response to resistance exercise. Studies have shown that at the beginning of resistance exercise, increase in stimulation of the sympathetic and parasympathetic activity and a decrease in plasma catecholamines transpire (Kenney et al., 2015). The increase in sympathetic activity can partially be related to the static resistance exercise and Valsalva manoeuvre conduction (Williams et al., 2007). During the created Valsalva manoeuvre, the rapid rise of pressure inside the aorta leads to a direct and immediate effect on arterial network which results in an increase in both systolic and diastolic blood pressure. Studies show that if the Valsalva manoeuvre is continued, the systolic and diastolic pressures reduce within seconds, which is due to the reduction in diastolic filling of the heart and its reason in turn is due to disorder in venous return (Mayo \& Kravitz, 1999). What seems evident in the effects of aerobic and resistance exercise in blood pressure, heart rate and myocardial oxygen cost, is that 15 to 60 minutes after aerobic and resistance exercise a significant reduction in the factors of cardiovascular occurs. Therefore, we can say that the continuation of such exercises in form of regular exercises will create good health and benefits in consecutive periods in line with cardiovascular health promotion of patients during rehabilitation. But, there were limitations uncontrolled by researchers such as: a) effectiveness of individual differences and genetic factors on the result of the study was not controlled, b) managing the level of motivation to apply maximum effort in the implementation of the tests was not possible, c) level of stress and psychological conditions in the subjects during the test and measurements was rampant.

\section{CONCLUSION}

According to the results of the study it could be concluded that aerobic and resistance exercise can affect blood levels of serotonin and dopamine in men with addiction, also it can reduce the harmful effects of methamphetamine on cardiovascular behaviour and be beneficial as a non-drug treatment. Thus, both aerobic and resistance exercises can be a valuable aid in the treatment of addiction and improvement of the addicted men's situation.

Authors' Contributions. All the authors were involved in study conceptualization, design, quality control and interpretation of the results. Hamid Arazi directed the fieldwork and drafted the 
manuscript. All the authors contributed to the final version of the manuscript.

Funding/Support. The present work was a master's thesis of exercise physiology and was partially supported by University of Guilan.
Conflict of Interests. None.

Acknowledgements. The authors would like to thank all the participants for their cooperation in this study and "Tarannom Ehyaye No" Institute for their support.

\section{REFERENCES}

Alberghina, D., Giannetto, C., \& Piccione, G. (2010). Peripheral serotoninergic response to physical exercise in athletic horses. Journal of Veterinary Science, 11(2), 285289. https://dx.doi.org/10.4142\%2Fjvs.2010.11.4.285

Andrews, C. M., \& Lucki, I. (2001). Effects of cocaine on extracellular dopamine and serotonin levels in the nucleus accumbens. Psychopharmacology, 155(4), 221229. https://doi.org/10.1007/s002130100704

Brown, B. S., Payne, T., Kim, C., Moore, G., Krebs, P., \& Martin, W. (1979). Chronic response of rat brain norepinephrine and serotonin levels to endurance training. Journal of Applied Physiology, 46(2), 19-23.

Brown, B. S., \& Van Huss, W. (1973). Exercise and rat brain catecholamines. Journal of Applied Physiology, 34(5), 664-669.

Brzycki, M. (1993). Strength testing-predicting a onerep max from reps-to-fatigue. Journal of Physical Education, Recreation \& Dance, 64(4), 88-90.

Dey, S., Singh, R., \& Dey, P. (1992). Exercise training: Significance of regional alterations in serotonin metabolism of rat brain in relation to antidepressant effect of exercise. Physiology \& Behavior, 52(3), 1095-1099.

Dolezal, B. A., Chudzynski, J., Dickerson, D., Mooney, L., Rawson, R. A., Garfinkel, A., \& Cooper, C. B. (2014). Exercise training improves heart rate variability after methamphetamine dependency. Medicine and Science in Sports and Exercise, 46(2), 1057. https://doi.org/10.1249/MSS.0000000000000201

Dwyer, D., \& Flynn, J. (2002). Short term aerobic exercise training in young males does not alter sensitivity to a central serotonin agonist. Experimental Physiology, 87(5), 83-89. doi: 10.1113/eph8702176

Floresco, S. B., Blaha, C. D., Yang, C.R. \& Phillips, A. G. (2001). Modulation of hippocampal and amygdalarevoked activity of nucleus accumbens neurons by dopamine: Cellular mechanisms of input selection. The Journal of Neuroscience, 21(9), 2851-2860.

Fontes-Ribeiro, C., Marques, E., Pereira, F., Silva, A., \& Macedo, T. (2011). May exercise prevent addiction? Current Neuropharmacology, 9(3), 45-48. https:// dx.doi.org/10.2174\%2F157015911795017380

George, J. D., Vehrs, P., Allsen, P. E., Fellingham, G. W., \& Fisher, A. G. (1993). VO $\sim 2 \sim \mathrm{m} \sim \mathrm{a} \sim \mathrm{x}$ estimation from a submaximal 1-mile track jog for fit collegeage individuals. Medicine and Science in Sports and Exercise, 25(4), 401-406.

Goekint, M., Bos, I., Heyman, E., Meeusen, R., Michotte, Y., \& Sarre, S. (2012). Acute running stimulates hippocampal dopaminergic neurotransmission in rats, but has no influence on brain-derived neurotrophic factor. Journal of Applied Physiology, 112(10), 535541. https://doi.org/10.1152/japplphysiol.00306.2011

Harvey, D. C., Lacan, G., Tanious, S. P., \& Melega, W. P. (2000). Recovery from methamphetamine induced longterm nigrostriatal dopaminergic deficits without substantia nigra cell loss. Brain Research, 871(8), 259-270.

Joanna, M., Małgorzata, F., \& Edmund, P. (2011). Role of serotonin (5-HT) 1B receptors in psychostimulant addiction. Pharmacological Reports, 63(6), 1310-1315. https://doi.org/10.1016/S1734-1140(11)70695-8

Jones, H., George, K., Edwards, B., \& Atkinson, G. (2007). Is the magnitude of acute post-exercise hypotension mediated by exercise intensity or total work done? European Journal of Applied Physiology, 102(4), 33-40.

Kenney, W. L., Wilmore, J., \& Costill, D. (2015). Physiology of sport \& exercise (6th Edition). Human Kinetics.

Langfort, J., Barańczuk, E., Pawlak, D., Chalimoniuk, M., Lukačova, N., Maršala, J., \& Górski, J. (2006). The effect of endurance training on regional serotonin metabolism in the brain during early stage of detraining period in the female rat. Cellular and Molecular Neurobiology, 26(2), 1325-1340.

Lohman, T., Roche, A., \& Martorell, R. (1991). Anthropometric standarization reference manual. Champaign, Ill.

Mayo, J. J. \& Kravitz, L. (1999). A review of the acute cardiovascular responses to resistance exercise of healthy young and older adults. The Journal of Strength \& Conditioning Research, 13(4), 90-96.

McCann, U. D., Kuwabara, H., Kumar, A., Palermo, M., Abbey, R., Brasic, J., ... Wong, D. F. (2008). Persistent cognitive and dopamine transporter deficits in abstinent methamphetamine users. Synapse, 62(5), 91-100.

Mohebbi, H., Rahmani-Nia, F., Vatani, D. S., \& Faraji, H. (2010). Post-exercise responses in blood pressure, heart rate and rate pressure product in endurance and resistance exercise. Medicina Dello Sport, 63(11), 209-219.

Mota, M. R., Pardono, E., Lima, L .C., Arsa, G., Bottaro, M., Campbell, C. S., \& Simões, H. G. (2009). Effects of treadmill running and resistance exercises on lowering blood pressure during the daily work of hypertensive subjects. The Journal of Strength \& Conditioning Research, 23(4), 2331-2338. https://doi. org/10.1519/JSC.0b013e3181bac418

O’dell, S. J., Galvez, B. A., Ball, A. J., \& Marshall, J. F. (2012). Running wheel exercise ameliorates methamphetamine - induced damage to dopamine and 
serotonin terminals. Synapse, 66(3), 71-80. https://doi. org/10.1002/syn.20989

Ricaurte, G. A., Schuster, C. R., \& Seiden, L. S. (1980). Long-term effects of repeated methylamphetamine administration on dopamine and serotonin neurons in the rat brain: A regional study. Brain Research, 193(5), 153-163.

Seiden, L. S., Sabol, K. E., \& Ricaurte, G. A. (1993). Amphetamine: Effects on catecholamine systems and behavior. Annual Review of Pharmacology and Toxicology, 33(2), 639-676. https://doi.org/10.1146/ annurev.pa.33.040193.003231

Spirduso, W.W., \& Farrar, R. P. (1981). Effects of aerobic training on reactive capacity: An animal model. Journal of Gerontology, 36(7), 654-662. https://doi.org/10.1093/ geronj/36.6.654

Tanaka, H., Monahan, K. D., \& Seals, D. R. (2001). Age-predicted maximal heart rate revisited. Journal of the American College of Cardiology, 37(7), 153-156.

Thompson, P. M., Hayashi, K. M., Simon, S. L., Geaga, J. A., Hong, M. S., Sui, Y., ... London, E. D. (2004). Structural abnormalities in the brains of human subjects who use methamphetamine. The Journal of Neuroscience, 24(9), 6028-6036.

Varner, K. J., Ogden, B. A., Delcarpio, J., \& MelegSmith, S. (2002). Cardiovascular responses elicited by the "binge" administration of methamphetamine. Journal of Pharmacology and Experimental Therapeutics, 301(8), 152-159.

Wang, G.-J., Volkow, N. D., Fowler, J. S., Franceschi, D., Logan, J., Pappas, N. R., Wong, C. T., \& Netusil, N. (2000). PET studies of the effects of aerobic exercise on human striatal dopamine release. Journal of Nuclear Medicine, 41(10), 1352-1356.

Wendt, M. (2002). Changing brain chemistry with intense exercise for drug addiction prevention and recovery, research paper presentation for treating addictions in special populations. Research Confronts Reality, 51(1), 7-8.

Williams, M. A., Haskell, W. L., Ades, P. A., Amsterdam, E. A., Bittner, V., Franklin, B. A., ... Stewart, K. J. (2007). Resistance exercise in individuals with and without cardiovascular disease: 2007 update a scientific statement from the american heart association council on clinical cardiology and council on nutrition, physical activity, and metabolism. Circulation, 116(12), 572-584. https://doi.org/10.1161/ CIRCULATIONAHA.107.185214

Zweben, J. E., Cohen, J. B., Christian, D., Galloway, G. P., Salinardi, M., Parent, D., \& Iguchi, M. (2004). Psychiatric symptoms in methamphetamine users. American Journal on Addictions, 13(3), 181-190. https://doi.org/10.1080/10550490490436055 